\title{
FATORES ASSOCIADOS À SATISFAÇÃO DO ACOMPANHANTE COM O CUIDADO PRESTADO À PARTURIENTE
}

\author{
Bruna Daniela Batista ${ }^{1}$, Odaléa Maria Bruggemann² ${ }^{2}$ Carolina Frescura Junges ${ }^{3}$, Manuela Beatriz Velho ${ }^{4}$, \\ Roberta Costa ${ }^{5}$
}

\begin{abstract}
RESUMO: O objetivo foi avaliar a satisfação do acompanhante com o cuidado prestado à mulher durante o trabalho de parto e nascimento e estimar fatores associados. Estudo transversal, realizado em Hospital Universitário no Sul do Brasil, foram entrevistados 369 acompanhantes de março a setembro 2015. As associações foram investigadas em análises bivariadas, razão de chance bruta e ajustada, através da regressão logística. A chance de satisfação foi maior nos acompanhantes que não presenciaram violência à mulher no trabalho de parto (RCaj 16,05; IC95\% $6,35-40,52$ ) e nascimento (RCaj 14,25; IC95\% 4,81-42,20), que acompanharam trabalho de parto que culminou em parto vaginal (RCaj 2,84; IC95\% 1,31-6,17), que consideraram que as vontades da mulher foram respeitadas (RCaj 8,16; IC95\% 3,41-19,54) e cujas queixas foram atendidas (RCaj 3,21; IC95\% 2,07-4,99). O relacionamento, atitudes e condutas dos profissionais de saúde influenciam na satisfação dos acompanhantes com o cuidado à mulher, independente das suas características sociodemográficas.
\end{abstract}

DESCRITORES: Parto humanizado; Enfermagem obstétrica; Avaliação de serviços de saúde; Assistência perinatal; Humanização da assistência.

\section{FACTORS ASSOCIATED WITH THE BIRTH COMPANION'S SATISFACTION WITH THE CARE PROVIDED TO THE PARTURIENT WOMAN}

ABSTRACT: The aim was to assess the birth companion's satisfaction with the care provided to the woman during labor and birth, and to calculate the associated factors. It is a cross-sectional study, undertaken in a University Hospital in the South of Brazil. In all, 369 companions were interviewed between March and September 2015. The associations were investigated with bivariate analyses and crude and adjusted odds ratio, using logistic regression. Satisfaction was most frequent in companions who did not witness violence to the woman during labor (adjOR 16.05; CI95\% 6.35-40.52) and birth (AdjOR 14.25; CI95\% 4.81-42.20), who were present during labor which culminated in vaginal birth (AdjOR 2.84; CI95\% 1.31-6.17), who considered that the woman's wishes were respected (AdjOR 8.16; CI95\% 3.41-19.54) and that her complaints were responded to (AdjOR 3.21; CI95\% 2.07-4.99). The health professionals' relationships, attitudes and conducts influenced the companions' satisfaction with the care provided to the woman, independently of their sociodemographic characteristics.

DESCRIPTORS: Humanizing delivery; Obstetric nursing; Health services research; Perinatal care; Humanization of Assistance.

\section{FACTORES ASOCIADOS A LA SATISFACCIÓN DEL ACOMPAÑANTE CON EL CUIDADO PREVENTIVO BRINDADO A LA PARTURIENTA}

RESUMEN: Se objetivó evaluar la satisfacción del acompañante con el cuidado brindado a la mujer durante el trabajo de parto y nacimiento, y estimar factores asociados. Estudio transversal, realizado en Hospital Universitario del Sur de Brasil. Fueron entrevistados 369 acompañantes de marzo a setiembre de 2015. Asociaciones investigadas por análisis bivariado, razón de probabilidades, ajustada por regresión logística. La posibilidad de satisfacción fue mayor en acompañantes que no presenciaron violencia a la mujer durante trabajo de parto (RCaj 16,05; IC95\% 6,35-40,52) y nacimiento (RCaj 14,25; IC95\% 4,81-42,20), que presenciaron trabajo de parto culminado en parto normal (RCaj 2,84; IC95\% 1,31-6,17), que consideraron que la voluntad de la mujer fue respetada (RCaj 8,16; IC95\% 3,41-19,54) y cuyas quejas fueron atendidas (RCaj 3,21; IC95\% 2,07-4,99). La relación actitudes y conducta de los profesionales de salud influyen en la satisfacción de los acompañantes respecto del cuidado a la mujer, independientemente de sus características sociodemográficas.

DESCRIPTORES: Parto Humanizado; Enfermería Obstétrica; Investigación en Servicios de Salud; Atención Perinatal; Humanización de la Atención.

${ }^{1}$ Enfermeira. Residente em Enfermagem Obstétrica. Maternidade Bairro Novo, Secretaria Municipal de Curitiba. Curitiba, PR, Brasil.

${ }^{2}$ Enfermeira. Doutora em Tocoginecologia. Docente do Programa de Pós-graduação em Enfermagem da Universidade Federal de Santa Catarina. Florianópolis, SC, Brasil.

${ }^{3}$ Enfermeira. Doutora em Enfermagem. Unidade Neonatal do Hospital Universitário da Universidade Federal de Santa Catarina. Florianópolis, SC, Brasil.

${ }^{4}$ Enfermeira. Doutora em Enfermagem. Serviço de Enfermagem do Centro Obstétrico do Hospital Universitário da Universidade Federal de Santa Catarina. Florianópolis, SC, Brasil.

${ }^{5}$ Enfermeira. Doutora em Enfermagem. Docente de Enfermagem e do Programa de Pós-graduação em Enfermagem da Universidade Federal de Santa Catarina. Florianópolis, SC, Brasil.

Autor Correspondente:

Recebido: $22 / 03 / 2017$

Odaléa Maria Bruggemann Finalizado: $12 / 07 / 2017$

Universidade Federal de Santa Catarina

R. Deputado Antônio Edu Vieira 1020 - 88040-001 - Florianópolis, SC, Brasil

E-mail: odalea.ufsc@gmail.com 


\section{INTRODUÇÃO}

Estudos têm mostrado que a presença de um acompanhante de escolha da mulher durante o trabalho de parto, parto e pós-parto imediato traz segurança e conforto à mulher deixando-a mais empoderada, calma e fortalecida para parir, o que auxilia na diminuição do tempo de trabalho de parto e das sensações dolorosas, reflete positivamente no Apgar do $5^{\circ}$ minuto de vida do recém-nascido e aumenta a satisfação delas com a experiência. Isto está relacionado ao fato de que ter um acompanhante presente aumenta a privacidade da mulher e diminui as intervenções desnecessárias ${ }^{(1-3)}$.

Destaca-se que desde 1996 a Organização Mundial da Saúde (OMS) recomenda, entre as práticas benéficas, a presença de um acompanhante de escolha da mulher durante o processo parturitivo ${ }^{(4)}$. A adesão às boas práticas é um fator determinante da humanização na atenção ao parto e nascimento, uma vez que melhora os desfechos maternos e neonatais ${ }^{(5-7)}$. Sabe-se também que a presença de um acompanhante contribui para a diminuição dos índices de violência obstétrica ${ }^{(8)}$.

Em 2005, com base nas recomendações da OMS, nas políticas públicas como o Programa de Humanização no Pré-natal e Nascimento, e apoiada pela Rede de Humanização do Nascimento, foi publicada a Lei $n^{\circ} 11.108$ que dispõe sobre o direito da mulher de ter um acompanhante durante o trabalho de parto, parto e pós-parto imediato nas instituições públicas e privadas ${ }^{(9-10)}$.

Pesquisas mostram que os acompanhantes se sentem satisfeitos com a experiência de vivenciar o processo parturitivo, se sentem úteis, e conseguem superar a insegurança, uma vez que não acreditavam conseguir passar por todo o processo. Além disso, reconhecem que sua presença foi importante para que a mulher vivenciasse o momento de forma mais tranquila ${ }^{(3,11)}$.

Contudo, a Pesquisa Nascer no Brasil mostrou que, apesar da Lei do acompanhante e de todos os benefícios relacionados a sua presença, apenas 18,8\% das mulheres entrevistadas contaram com a presença do acompanhante em todos os momentos da internação ${ }^{(2)}$. No entanto, foi possível identificar que a sua presença diminui desigualdades sociais no atendimento, e melhora a percepção das mulheres sobre o atendimento recebido. Mulheres com acompanhante são mais respeitadas, relatam maior privacidade, sofrem menos violência, possuem maior possibilidade para fazer perguntas e participar das decisões ${ }^{(8)}$.

No contexto brasileiro, a presença do acompanhante durante o trabalho de parto, parto e pósparto imediato está garantida por Lei, sendo assim, muitas instituições de saúde passaram a permitir que ele participe desses momentos junto à mulher. No entanto, a maioria das pesquisas sobre essa prática é baseada na percepção das mulheres e dos profissionais, tornando-se necessária a realização de investigações que permitam que o acompanhante expresse como foi a sua experiência ao lado da parturiente ${ }^{(1,8)}$. Com isso, ele se torna um potencial informante sobre aspectos da assistência obstétrica ainda não revelados. Desta forma, o objetivo deste estudo foi avaliar a satisfação do acompanhante com o cuidado prestado à mulher e estimar fatores associados.

\section{- MÉTODO}

Pesquisa transversal inserida no macroprojeto "A participação do acompanhante de escolha da mulher no pré-natal, trabalho de parto e parto no sistema de saúde público".

Os dados foram coletados no alojamento conjunto de um Hospital Universitário do Sul do Brasil. A amostra foi calculada com base no número de nascimentos ocorridos na referida instituição no ano de 2013, que totalizou 1.525 nascimentos. A satisfação dos acompanhantes foi estimada em 50\%, com intervalo de confiança de $95 \%$ e erro máximo de 5\%. Amostra mínima calculada resultou em 307 acompanhantes, foram entrevistados 369 acompanhantes elegíveis entre março e setembro de 2015.

Os critérios de inclusão foram ter sido acompanhante de mulher que teve parto vaginal ou cesariana, de gestação única, que entraram em trabalho de parto e que o parto tenha ocorrido há mais de 4 horas, nas dependências da maternidade. Foram excluídos os acompanhantes de mulheres que não tenham entrado em trabalho de parto, e também de mulheres que foram a óbito, ou cujo recém-nascido tenha ido a óbito. 
Os dados foram coletados por meio de entrevista, para as quais foi criado um software e instalado em netbooks de uso exclusivo da pesquisa. O questionário foi testado previamente e as questões inconsistentes e de difícil compreensão foram reestruturadas e testadas novamente.

O questionário utilizado no macroprojeto é composto por seções que contemplam perguntas sobre a participação do acompanhante desde o pré-natal até o pós-parto.

O desfecho investigado foi a satisfação com o cuidado prestado à mulher durante o trabalho de parto e nascimento (parto vaginal/cesariana). A variável satisfação do acompanhante foi avaliada a partir de perguntas ao acompanhante sobre como se sentiu em relação à forma como cuidaram da mulher durante o trabalho de parto, parto e cesariana (muito insatisfeito, insatisfeito, satisfeito, bem satisfeito e muito satisfeito). Considerou-se como indicativo de satisfação o agrupamento das respostas "bem satisfeito e muito satisfeito" ${ }^{\prime 12-13)}$.

As variáveis preditoras foram: participação em curso/palestra durante o pré-natal, tipo de parto acompanhado (parto vaginal, cesariana), as relacionadas com o atendimento prestado à mulher, e ter presenciado violência à mulher (verbal, física, psicológica).

Os dados foram analisados no programa Stata/SE versão 13. As associações entre as variáveis preditoras e o desfecho foram investigadas em análises bivariadas, com estimação da Razão de Chance (RC) bruta e ajustada, através da Regressão Logística. As variáveis idade, sexo, escolaridade, participação em curso/palestra no pré-natal e tipo de parto foram consideradas fatores de confusão.

O macroprojeto, em que a pesquisa está inserida, foi aprovado pelo Comitê de Ética e Pesquisa com Seres Humanos da Universidade Federal de Santa Catarina, via Plataforma Brasil, parecer de número 541.296. Todos os acompanhantes assinaram o Termo de Consentimento Livre e Esclarecido.

\section{RESULTADOS}

Foram realizadas 369 entrevistas com os acompanhantes da mulher, que participaram do trabalho de parto e nascimento. A Tabela 1 apresenta as características sociodemográficas e experiências anteriores dos acompanhantes, sendo $82,66 \%$ (305) deles do sexo masculino, $42,82 \%$ (158) com idade entre 26 e 35 anos e 68,56\% (253) sem experiência anterior como acompanhante.

Tabela 1 - Satisfação do acompanhante segundo variáveis sociodemográficas, informação sobre a lei do acompanhante e experiência anterior. Florianópolis, SC, Brasil, 2015 (N=369) (continua)

\begin{tabular}{|c|c|c|c|c|c|c|}
\hline & \multicolumn{6}{|c|}{ Satisfação com o cuidado prestado à mulher } \\
\hline & \multirow[b]{2}{*}{$\mathbf{n}$} & \multirow[b]{2}{*}{$\%($ IC 95\%) } & \multicolumn{2}{|c|}{ Trabalho de parto } & \multicolumn{2}{|c|}{$\begin{array}{c}\text { Nascimento (Parto vaginal/ } \\
\text { cesariana) }\end{array}$} \\
\hline & & & $\mathbf{N}$ & $\%($ IC 95\%) & $\mathbf{n}$ & $\%($ IC 95\%) \\
\hline \multicolumn{7}{|l|}{ Idade } \\
\hline 18 a 25 & 96 & $26,02(21,78-30,76)$ & 85 & $88,54(80,40-93,57)$ & 88 & $91,67(84,13-95,80)$ \\
\hline 26 a 35 & 158 & $42,82(37,84-47,95)$ & 147 & $93,04(87,82-96,12)$ & 150 & $94,94(90,16-97,46)$ \\
\hline 36 a 45 & 70 & $18,97(15,27-23,32)$ & 65 & $92,86(83,83-97,02)$ & 68 & $97,14(89,14-99,29)$ \\
\hline 46 a 69 & 45 & $12,20(9,22-15,97)$ & 41 & $91,11(78,34-96,67)$ & 44 & $97,78(85,48-99,70)$ \\
\hline \multicolumn{7}{|l|}{ Sexo } \\
\hline Masculino & 305 & $82,66(78,43-86,20)$ & 278 & $91,15(87,38-93,87)$ & 288 & $94,43(91,20-96,52)$ \\
\hline Feminino & 64 & $17,34(13,80-21,57)$ & 60 & $93,75(84,35-97,66)$ & 62 & $96,88(88,19-99,23)$ \\
\hline \multicolumn{7}{|l|}{ Vínculo com a mulher } \\
\hline $\begin{array}{l}\text { Companheiro/pai do } \\
\text { bebê }\end{array}$ & 306 & $82,93(78,72-86,45)$ & 279 & $91,18(87,42-93,89)$ & 289 & $94,44(91,23-96,53)$ \\
\hline Mãe & 42 & $11,38(8,51-15,06)$ & 39 & $92,86(79,78-97,72)$ & 41 & $97,62(84,51-99,68)$ \\
\hline Outros* & 21 & $5,69(3,73-8,58)$ & 20 & $95,24(71,73-99,37)$ & 20 & $95,24(71,73-99,37)$ \\
\hline
\end{tabular}


Cogitare Enferm. (22)3: e51355, 2017

\begin{tabular}{|c|c|c|c|c|c|c|}
\hline \multicolumn{7}{|c|}{ Experiência anterior como acompanhante no trabalho de parto } \\
\hline Não & 253 & $68,56(63,62-73,12)$ & 229 & $90,51(86,21-93,57)$ & 239 & $94,47(90,85-96,70)$ \\
\hline Sim & 116 & $31,44(26,88-36,38)$ & 109 & $93,97(87,82-97,11)$ & 111 & $95,69(90-98,20)$ \\
\hline \multicolumn{7}{|c|}{ Experiência anterior como acompanhante em parto normal } \\
\hline Não & 289 & $78,32(73,80-82,25)$ & 263 & $91(87,09-93,81)$ & 274 & $94,81(91,55-96,86)$ \\
\hline Sim & 80 & $21,68(17,75-26,20)$ & 75 & $93,75(85,74-97,40)$ & 76 & $95(87,32-98,13)$ \\
\hline \multicolumn{7}{|c|}{ Experiência anterior como acompanhante em cesariana } \\
\hline Não & 329 & $89,16(85,54-91,96)$ & 302 & $91,79(88,28-94,32)$ & 312 & $94,83(91,83-96,77)$ \\
\hline Sim & 40 & $10,84(8,04-14,46)$ & 36 & $90(75,90-96,26)$ & 38 & $95(81,75-98,77)$ \\
\hline \multicolumn{7}{|c|}{ Informação sobre a Lei do acompanhante } \\
\hline Não & 290 & $78,59(74,08-82,50)$ & 267 & $92,07(88,33-94,68)$ & 275 & $94,83(91,58-96,87)$ \\
\hline Sim & 79 & $21,41(17,50-25,91)$ & 71 & $89,87(80,93-94,89)$ & 75 & $94,94(87,17-98,10)$ \\
\hline
\end{tabular}

$\mathrm{Na}$ análise ajustada, permaneceram como fatores associados à satisfação do acompanhante com cuidado prestado à mulher no trabalho de parto: não ter participado de curso/palestra no pré-natal, achar que o local de trabalho de parto era adequado, acompanhar parto vaginal e não presenciar nenhum tipo de violência. Também foram identificados como fatores associados considerar que: os profissionais respeitaram as vontades da mulher; solucionaram as queixas dela; estavam disponíveis para atender as demandas; explicaram o que estava acontecendo durante o trabalho de parto de forma clara e compreensível; ofereceram alternativas para diminuir a dor durante trabalho de parto (Tabela 2).

Tabela 2 - Razão de chance (RC) bruta e ajustada para o desfecho satisfação do acompanhante segundo palestra/ curso no pré-natal, tipo de parto e variáveis relacionadas ao atendimento prestado à mulher durante trabalho de parto e ocorrência de violência. Florianópolis, SC, Brasil, 2015 ( $N=369)$ (continua)

\begin{tabular}{|c|c|c|c|c|c|c|}
\hline & \multicolumn{6}{|c|}{ Satisfação quanto ao cuidado prestado à mulher } \\
\hline & \multicolumn{2}{|c|}{ Trabalho de parto } & \multicolumn{4}{|c|}{ Nascimento } \\
\hline & RC (IC 95\%) & RCaj* (IC 95\%) & $\begin{array}{c}\text { Valor de } \\
\text { p** }^{* *} \\
\end{array}$ & RC (IC 95\%) & RCaj* (IC 95\%) & $\begin{array}{c}\text { Valor } \\
\text { de } \mathbf{p}^{* *}\end{array}$ \\
\hline \multicolumn{7}{|c|}{ Participação em curso/palestra no pré-natal } \\
\hline Sim & Referência & & & Referência & & \\
\hline Não & $2,32(0,98-5,52)$ & $2,86(1,13-7,27)$ & 0,027 & $3,05(1,10-8,42)$ & $2,61(0,88-7,71)$ & 0,082 \\
\hline \multicolumn{7}{|c|}{ Profissionais respeitaram as vontades da mulher } \\
\hline Não & Referência & & & Referência & & \\
\hline Sim & $8,67(3,83-19,64)$ & $8,16(3,41-19,54)$ & $<0,001$ & $9,6(3,62-25,46)$ & $8,89(3,19-24,74)$ & $<0,001$ \\
\hline \multicolumn{7}{|c|}{ Profissionais solucionaram as queixas da mulher } \\
\hline Não & Referência & & & Referência & & \\
\hline Sim & $3,22(2,12-4,89)$ & $3,21(2,07-4,99)$ & $<0,001$ & $2,59(1,56-4,29)$ & $2,43(1,45-4,09)$ & 1 \\
\hline \multicolumn{7}{|c|}{ Profissionais disponíveis para atender as demandas da mulher } \\
\hline Não & Referência & & & Referência & & \\
\hline Sim & $1,98(1,61-2,43)$ & $1,88(1,51-2,34)$ & $<0,001$ & $1,78(1,39-2,27)$ & $1,76(1,35-2,28)$ & $<0,001$ \\
\hline \multicolumn{7}{|c|}{ Profissionais deram explicações para a mulher sobre o TP*** } \\
\hline Não & Referência & & & Referência & & \\
\hline Sim & $1,77(1,47-2,12)$ & $1,74(1,42-2,14)$ & $<0,001$ & $1,53(1,24-1,90)$ & $1,53(1,21-1,93)$ & $<0,001$ \\
\hline \multicolumn{7}{|c|}{ As informações foram claras e compreensíveis } \\
\hline Não & Referência & & & Referência & & \\
\hline Sim & $1,48(1,28-1,71)$ & $1,51(1,29-1,76)$ & $<0,001$ & $1,48(1,25-1,74)$ & $1,44(1,21-1,71)$ & $<0,001$ \\
\hline
\end{tabular}




\begin{tabular}{|c|c|c|c|c|c|c|}
\hline \multicolumn{7}{|c|}{ Ofertaram alternativas para diminuir dor e desconforto da mulher durante TP*** } \\
\hline \multirow{2}{*}{$\begin{array}{l}\text { Não } \\
\text { Sim }\end{array}$} & \multirow{2}{*}{$\frac{\text { Referência }}{1,20(1,08-1,35)}$} & \multicolumn{5}{|c|}{ Referência } \\
\hline & & $1,19(1,05-1,34)$ & 0,005 & $1,14(0,99-1,31)$ & $1,14(0,98-1,33)$ & 0,082 \\
\hline \multicolumn{7}{|c|}{ Local que vivenciou TP*** e nascimento era adequado } \\
\hline Não & Referência & & & Referência & & \\
\hline Sim & $1,22(1,09-1,37)$ & $1,25(1,10-1,43)$ & $<0,001$ & $1,26(1,10-1,43)$ & $1,23(1,07-1,41)$ & 0,004 \\
\hline \multicolumn{7}{|c|}{ Violência (verbal, física, psicológica) } \\
\hline Não & Referência & & & Referência & & \\
\hline Sim & $14,61(6,42-33,23)$ & $16,05(6,35-40,52)$ & $<0,001$ & $12,30(4,63-32,69)$ & $14,25(4,81-42,20)$ & $<0,001$ \\
\hline \multicolumn{7}{|c|}{ Tipo de parto } \\
\hline Cesariana & Referência & & & Referência & & \\
\hline Normal & $2,75(1,30-5,79)$ & $2,84(1,31-6,17)$ & 0,008 & $0,94(0,33-2,69)$ & $1,05(0,36-3,05)$ & 0,932 \\
\hline
\end{tabular}

*RCaj - Razão de Chance ajustada para idade, sexo, escolaridade, curso/palestra no pré-natal e tipo de parto. **Nível de significância pelo Teste de Wald. ***TP - Trabalho de Parto

Quanto à satisfação do acompanhante com cuidado prestado durante o nascimento, na análise ajustada, foram fatores associados: profissionais respeitarem as vontades da mulher; solucionarem as queixas e atenderam as demandas dela, darem explicações para a mulher; informações claras e compreensíveis; quando o local do nascimento era adequado; e quando o acompanhante não presenciou violência verbal, física ou psicológica (Tabela 2).

\section{- DISCUSSÃO}

A amostra estudada revela que a maioria dos acompanhantes era do sexo masculino e companheiro/ pai do bebê, achado semelhante ao de outras pesquisas realizadas no Brasil(2-3,14-15). Alguns estudos mostram que, dependendo do contexto social, nem sempre essa é a escolha mais frequente da mulher, uma vez que a mãe da parturiente em algumas maternidades é a mais presente ${ }^{(16-17)}$. Assim, este estudo evidencia que cada vez mais a livre escolha da mulher por um acompanhante está sendo respeitada ${ }^{(18)}$, e que a inserção da figura paterna tem sido a mais frequente ${ }^{(2-3,14,19)}$. Esse fato contribui para o fortalecimento de laços entre pai e filho e, muitas vezes, entre o próprio casal, formando a tríade mãe-pai-filho ${ }^{(3,20-21)}$.

Apesar da Lei do Acompanhante existir há 11 anos, a maioria dos entrevistados referiram não terem recebido informação sobre ela, assim como evidenciado em outras pesquisas ${ }^{(11,17)}$. Isto pode ser um reflexo de uma assistência pré-natal voltada para os aspectos clínicos, não priorizando o cumprimento do $10^{\circ}$ passo para uma assistência pré-natal de qualidade - informar a mulher quanto aos seus direitos garantidos por lei, segundo o Ministério da Saúde $(\mathrm{MS})^{(22)}$, uma vez que a informação é o que permite que mulheres e acompanhantes cheguem à maternidade melhor preparados para o processo parturitivo.

Não ter informação sobre a lei é algo preocupante, entretanto, isso parece não ser um impeditivo para a participação do acompanhante de escolha da mulher na maternidade estudada, uma vez que a inserção desse personagem se dá desde a implantação do serviço em $1995^{(18,23)}$. Vale ressaltar que os resultados aqui analisados mostram que a maioria dos entrevistados estava vivenciando o papel de acompanhante pela primeira vez, assim como mostram outros estudos ${ }^{(3,11)}$.

Os resultados mostram que a idade, escolaridade, vínculo com a mulher e experiências anteriores como acompanhante não foram associadas ao desfecho. A chance de satisfação foi maior para os acompanhantes que estiveram junto à mulher durante o trabalho de parto que evoluiu para um parto vaginal, e quando consideraram que os profissionais respeitaram as vontades da mulher, solucionaram as queixas, forneceram explicações à mulher sobre o trabalho de parto, repassaram informações claras e compreensíveis; ofereceram métodos para alívio da dor e desconforto no trabalho de parto; e que avaliaram o ambiente como adequado. 
A chance do acompanhante ficar satisfeito com o cuidado prestado à mulher durante o trabalho de parto foi duas vezes maior entre aqueles que não participaram de curso/palestra, contudo não manteve associação com a satisfação durante o nascimento. Estes achados sugerem que o acompanhante que possui informação sobre o processo parturitivo e sobre a assistência prestada na maternidade consegue ter parâmetros para avaliar o atendimento, sabendo distinguir com maior facilidade se as práticas realizadas foram adequadas ou não.

Informar o acompanhante quanto às diversas ações desenvolvidas dentro de um centro obstétrico, aumenta a sua autonomia e o fortalece para questionar sobre o atendimento prestado(24). Ter informações previamente sobre o processo parturitivo e sobre a assistência prestada na maternidade permite que o acompanhante crie expectativas acerca de um padrão de qualidade de atendimento e, portanto, quando esse não é alcançado, a chance de satisfação diminui( ${ }^{(25)}$. Estudo com puérperas também mostrou que a atitude profissional é determinante na satisfação com a experiência ${ }^{(8)}$.

Destaca-se que os acompanhantes de mulheres que tiveram parto vaginal tiveram duas vezes maior chance de se sentirem satisfeitos com o cuidado prestado à mulher durante o trabalho de parto, quando comparados aos que acompanharam cesariana. O trabalho de parto é considerado um processo de transição, tanto para a mulher que se tornará mãe, quanto para o homem que se tornará pai $^{(26-27)}$. Sendo assim, este momento é visto pelo pai-acompanhante como um processo doloroso para a mulher, porém necessário para que se atinja a vitória, que é representada pelo nascimento ${ }^{(27)}$. Portanto, pode-se inferir que o acompanhante que experiencia o parto vaginal fica mais satisfeito com o cuidado prestado à mulher durante o trabalho parto, por ter contribuído de forma efetiva para o alcance do desfecho esperado.

Cabe destacar que a maternidade estudada, desde sua implantação em 1995, possui como filosofia a humanização e interdisciplinaridade, tendo como princípios a inclusão da família no processo gestacional e parturitivo, bem como um atendimento personalizado, baseado em evidências científicas $^{(23)}$.

Os acompanhantes que não presenciaram nenhum tipo de violência (verbal, física ou psicológica) tiveram 16 vezes maior chance de se sentir satisfeito com relação à forma que cuidaram da mulher no trabalho de parto, e 14 vezes maior chance de se sentir satisfeito com o cuidado no nascimento. Os achados deste estudo mostram que os acompanhantes conseguem avaliar a forma como a mulher foi assistida e que, apesar dos benefícios comprovados, a sua presença não foi capaz de inibir totalmente as atitudes dos profissionais que caracterizam a violência verbal, física e psicológica.

Na Pesquisa Nascer no Brasil, ter o acompanhante em todos os momentos foi associado à menor chance das mulheres sofrerem violência e pode estimular uma relação mais cordial por parte dos profissionais de saúde ${ }^{(8)}$. O momento do parto e nascimento é de extrema importância não apenas na vida da mulher, mas também de seu acompanhante, e presenciar algum tipo de violência se mostra como fator importante na insatisfação de ambos com a experiência.

O acompanhante que percebeu que os profissionais respeitaram as vontades da mulher teve oito vezes maior chance de estar satisfeito com o cuidado prestado, tanto no trabalho de parto quanto no nascimento. Além disso, quando as queixas da mulher eram solucionadas, a chance de satisfação do acompanhante com o cuidado triplicou. Isso mostra que o acompanhante está emocionalmente envolvido com o processo compartilhado com a mulher ${ }^{(11)} \mathrm{e}$, desta forma, ele se torna um sujeito importante na avaliação da assistência prestada nas maternidades.

Esses achados são congruentes com as recomendações acerca de como deve ser estabelecida a relação do profissional com a parturiente, contempladas nas Diretrizes nacionais de assistência ao parto normal: os profissionais de saúde devem respeitar as mulheres em trabalho de parto e estabelecer uma relação igualitária, perguntando-lhes sobre seus desejos e expectativas. Além disso, devem considerar a importância de sua atitude, da forma como se comunicam com as mulheres, bem como com os cuidados prestados ${ }^{(28)}$.

Aspectos semelhantes foram apontados em estudo sobre a avaliação das mulheres acerca do atendimento ao parto, nos quais a relação com o profissional, a clareza nas explicações recebidas e a possibilidade de fazer perguntas foram fatores associados com a satisfação ${ }^{(8)}$. Envolver a mulher e seu acompanhante na tomada de decisão é uma das formas de trazer qualidade e humanização à atenção 
obstétrica ${ }^{(28)}$ e, assim, fazê-los se sentirem parte ativa do processo e com isso, aumentar a satisfação com a experiência.

Foi possível identificar que condutas inadequadas como a violência à mulher que está vivenciando o trabalho de parto, influenciaram negativamente na satisfação do acompanhante. Por outro lado, atitude respeitosa, condutas resolutivas, informações claras e compreensíveis, e local adequado foram fatores associados com a satisfação do acompanhante.

Destaca-se como limitação desse estudo, o fato de as entrevistas terem sido realizadas durante a internação da mulher, o que pode gerar um viés de cortesia, que pode ter influenciado nas respostas dos acompanhantes.

\section{CONCLUSÃO}

O respeito, o fornecimento de explicações claras e compreensíveis, o atendimento às queixas da mulher e a disponibilidade dos profissionais para atender às suas demandas, são fatores associados à satisfação do acompanhante.

Pesquisas acerca da satisfação dos acompanhantes ainda são escassas, demonstrando a necessidade de mais estudos que envolvam esta temática. Contudo, é interessante ressaltar que a maioria dos achados deste estudo é semelhante aos realizados com puérperas, indicando que a mulher e acompanhante estão conectados emocionalmente e compartilham ideias e opiniões sobre a experiência.

O elevado percentual de acompanhantes satisfeitos com o cuidado prestado à mulher pode ser decorrente do próprio contexto em que a pesquisa foi desenvolvida, uma vez que a maternidade possui uma filosofia com princípios de humanização da assistência, o que pode ter gerado um viés nos resultados. Dessa forma, se torna imprescindível avaliar a satisfação dos acompanhantes em outros contextos assistenciais.

Com este estudo, é possível inferir que o relacionamento, as atitudes e condutas dos profissionais de saúde são capazes de influenciar na satisfação dos acompanhantes sobre o modo como cuidam da mulher, uma vez que eles experienciam com ela todo o processo parturitivo.

\section{- AGRADECIMENTO}

Ao Conselho Nacional de Desenvolvimento Científico e Tecnológico (CNPq) pelo financiamento concedido para a realização da pesquisa, através da Chamada Pública MCTI/CNPq No 14/2013 Universal, Processo: 473810/2013-1.

\section{REFERÊNCIAS}

1. Hodnett ED, Gates S, Hofmeyr GJ, Sakala C. Continuous support for women during childbirth. Cochrane Database Syst Rev. [Internet] 2013;(7) [acesso em 12 fev 2017]. Disponível: http://dx.doi.org/10.1002/14651858. CD003766.pub5.

2. Diniz CSG, D'Orsi E, Domingues RMSM, Torres JA, Dias MAB, Schneck CA, et al. Implementação da presença de acompanhantes durante a internação para o parto: dados da pesquisa nacional Nascer no Brasil. Cad. Saúde Pública. [Internet] 2014;30(Suppl 1) [acesso em 24 mai 2016]. Disponível: http://dx.doi.org/10.1590/0102311X00127013.

3. Brüggemann OM, Koettker JG, Velho MB, Monguilhott JJC, Monticelli M. Satisfação dos acompanhantes com a experiência de apoiar a parturiente em um hospital universitário. Texto Contexto Enferm. [Internet] 2015;24(3) [acessoem 07 abr 2016]. Disponível: http://dx.doi.org/10.1590/0104-07072015004220014.

4. Organização Mundial da Saúde (OMS). Assistência ao parto normal:um guia prático. Genebra: Organização Mundial de Saúde; 1996. 
5. Lansky S, Friche AAL, da Silva AAM, Campos D, Bittencourt SDA, de Carvalho ML, et al. Pesquisa Nascer no Brasil: perfil da mortalidade neonatal e avaliação da assistência à gestante e ao recém-nascido. Cad. Saúde Pública. [Internet] 2014;30(Suppl 1) [acesso em 23 mai 2016]. Disponível: http://dx.doi.org/10.1590/0102-311X00133213.

6. Leal MC, Pereira APE, Domingues RMSM, Theme Filha MM, Dias MAB, Nakamura-Pereira M, et al. Intervenções obstétricas durante o trabalho de parto e parto em mulheres brasileiras de risco habitual. Cad. Saúde Pública. [Internet] 2014;30(Suppl 1) [acesso em 01 mar 2016]. Disponível: http://dx.doi.org/10.1590/0102-311X00151513.

7. Andrade PON, da Silva JQP, Diniz CMM, Caminha MFC. Fatores associados à violência obstétrica na assistência ao parto vaginal em uma maternidade de alta complexidade em Recife, Pernambuco. Rev. Bras. Saude Mater. Infant. [Internet] 2016;16(1) [acesso em 23 maio 2016]. Disponível: http://dx.doi.org/10.1590/1806-93042016000100004.

8. D'Orsi E, Brüggemann OM, Diniz CSG, de Aguiar JM, Gusman CR, Torres JA, et al. Desigualdades sociais e satisfação das mulheres com o atendimento ao parto no Brasil: estudo nacional de base hospitalar. Cad. Saúde Pública. [Internet] 2014;30(Suppl 1) [acesso em 23 jun 2015]. Disponível: http://dx.doi.org/10.1590/0102311X00087813.

9. Brasil. Lei n. 11.108. de 7 de abril de 2005. Altera a Lei n. 8.080, de 19 de setembro de 1990: dispões assim sobre a garantia às parturientes o direito à presença de acompanhante durante o trabalho de parto, parto e pós-parto imediato, no âmbito do Sistema Único de Saúde - SUS. Diário Oficial da União da República Federativa do Brasil, Brasília, 8 abr 2005. Seção 1:1.

10. Rattner D, dos Santos ML, Lessa H, Diniz SG. ReHuNa: A Rede pela Humanização do Parto e Nascimento. Rev Tempus Actas: Saúde Col. [Internet] 2010;4(4) [acesso em 26 mai 2016]. Disponível:http://www.tempusactas.unb. br/index.php/tempus/article/view/849/812.

11. Frutuoso LD, Brüggemann OM. Conhecimento sobre a Lei 11.108/2005 e a experiência dos acompanhantes junto à mulher no centro obstétrico. Texto Contexto Enferm. [Internet] 2013;22(4) [acesso em 24 mai 2016 ]. Disponível: http://dx.doi.org/10.1590/S0104-07072013000400006.

12. Brown S, Lumley J. Satisfaction with care in labor and birth: a survey of 790 Australian women. Birth. [Internet] 1994;21(1) [acesso em 12 fev 2017]. Disponível: http://dx.doi.org/10.1111/j.1523-536X.1994.tb00909.x.

13. Sadler LC, Davison T, McCowan LME. Maternal satisfaction with active management of labor: A randomized controlled trial. Birth. [Internet] 2001;28(4) [acesso em 12 fev 2017]. Disponível: http://dx.doi.org/10.1046/j.1523536X.2001.00225.x.

14. Ministério da Saúde (BR). Relatório Preliminar de Pesquisa: Resultados preliminares da Pesquisa de Satisfação com mulheres puérperas atendidas no Sistema Único de Saúde - SUS. Maio de 2012 a fevereiro de 2013. Brasília: Secretaria de Gestão Estratégica e Participativa; Departamento de Ouvidoria Geral do SUS; Ministério da Saúde; 2013.

15. Gonçalves AC, da Rocha CM, Gouveia HG, Armellini CJ, Moretto VL, Moraes BA. O acompanhante no centro obstétrico de um hospital universitário do sul do Brasil. Rev. Gaúcha Enferm. [Internet] 2015;36(n.esp) [acesso em 09 jun 2016]. Disponível: http://dx.doi.org/10.1590/1983-1447.2015.esp.57289.

16. Hotimsky SN, Alvarenga AT. A definição do acompanhante no parto: uma questão ideológica?. Rev. Estud. Fem. [Internet] 2002;10(2) [acesso em 24 mai 2016]. Disponível: http://dx.doi.org/10.1590/S0104-026X2002000200015.

17.de Oliveira AS, Damasceno AKC, de Moraes JL, Moreira KAP, Teles LMR, Gomes LFS. Technology used by companions in labor and childbirth: a descriptive study. Online Braz J Nurs. [Internet] 2014;13(1) [acesso em 26 mai 2016]. Disponível: http://www.objnursing.uff.br/index.php/nursing/article/view/4254.

18. Brüggemann OM, Monticelli M, Furtado C, Fernandes CM, Lemos FN, Gayeski ME. Filosofia assistencial de uma maternidade-escola: fatores associados à satisfação das mulheres usuárias. Texto Contexto Enferm. [Internet] 2011;20(4) [acesso em 24 mai 2016]. Disponível: http://dx.doi.org/10.1590/S0104-07072011000400003.

19. Dodou HD, Rodrigues DP, Guerreiro EM, Guedes MVC, do Lago PN, de Mesquita NS. A contribuição do acompanhante para a humanização do parto e nascimento: percepções de puérperas. Esc. Anna Nery. [Internet] 2014;18(2) [acesso em 09 jun 2016]. Disponível: http://dx.doi.org/10.5935/1414-8145.20140038.

20. Carvalho IS, da Costa Júnior PB, Macedo JBPO, de Araújo RDT. Acompanhantes no processo de nascimento: benefícios reconhecidos pelos enfermeiros. J Health Sci Inst. [Internet] 2013;31(2) [acesso em 29 mai 2016]. 
Disponível: http://www.unip.br/comunicacao/publicacoes/ics/edicoes/2013/02_abr-jun/V31_n2_2013_p166a171. pdf.

21. de Oliveira AG, Silva RR. Parto também é assunto de homens: Uma pesquisa clínico-qualitativa sobre a percepção dos pais acerca de suas reações psicológicas durante o parto. Interação Psicol. [Internet] 2012;16(1). [acesso em 12 fev 2017]. Disponível: http://dx.doi.org/10.5380/psi.v16i1.22970.

22. Ministério da Saúde (BR). Secretaria de Atenção à Saúde. Departamento de Atenção Básica. Atenção ao prénatal de baixo risco. Nº 32. Brasília: Ministério da Saúde; 2012.

23. Santos OMB, Siebert ERC. The humanization of birth experience at the University of Santa Catarina maternity hospital. Int J Gynaecol Obstet. [Internet] 2001;75(Suppl 1) [acesso em 04 jun 2016]. Disponível:http://doi. org/10.1016/S0020-7292(01)00514-8.

24. Basso JF, Monticelli M. Expectativas de participação de gestantes e acompanhantes para o parto humanizado. Rev. Latino-Am. Enfermagem. [Internet] 2010;18(3) [acesso em 12 jun 2016]. Disponível: http://dx.doi.org/10.1590/ S0104-11692010000300014.

25. Murray CJL, Kawabata K, Valentine N. People's Experience Versus People's Expectations. Health Affairs. [Internet] 2001;20(3) [acesso em 12 jun 2016]. Disponível: http://dx.doi.org/10.1377/hlthaff.20.3.21.

26. Velho MB, dos Santos EKA, Brüggemann OM, Camargo BV. Vivência do parto normal ou cesáreo: revisão integrativa sobre a percepção de mulheres. Texto Contexto Enferm. [Internet] 2012;21(2) [acesso em 12 jun 2016]. Disponível: http://dx.doi.org/10.1590/S0104-07072012000200026.

27. Jardim DMB, Penna CMM. Pai-acompanhante e sua compreensão sobre o processo de nascimento do filho. Reme, Rev. Min. Enferm. [Internet] 2012;16(3) [acesso em 12 jun 2016]. Disponível: http://www.reme.org.br/artigo/ detalhes/540.

28. Ministério da Saúde (BR). Diretrizes Nacionais de Assistência ao Parto Normal. Conselho Nacional de Incorporação de Tecnologias no SUS. Brasília: Secretaria de Ciência, Tecnologia e Insumos Estratégicos; Ministério da Saúde; 2016. 